\title{
Contextos de Aprendizagem para Interações Contingentes em Fóruns de Discussão Online: uma Investigação à Luz da Etnografia Interacional
}

\author{
Priscila Barros David ${ }^{1}$, Judith L. Green ${ }^{2}$, Vanessa Ellen Cacau dos Santos ${ }^{1}$ \\ Universidade Federal do Ceará (UFC) - Instituto Universidade Virtual \\ Bloco Acadêmico - Campus do Pici, s/n, Bloco 1430 - Fortaleza-CE - CEP 60.440-900 \\ University of California, Santa Barbara (UCSB), Department of Education, Gevirtz \\ Graduate School of Education, Santa Barbara, California/USA - 93106 \\ priscila@virtual.ufc.br, judithlgreen@me.com, vanessacacaus@gmail.com
}

\begin{abstract}
This study aims to identify and characterize the learning contexts favorable to the implementation of contingent interactions in the discussion forum of an Online Tutors Formation Program. The Interactional Ethnographic approach that guided this research established four operational principles that guide ethnographer's actions and decicions in the field of investigation. Such principles promoted a broader understanding of learning contexts in the forum. The results of the study pointed out, mainly: the fragility of the interaction representation mechanism in the investigated tool and the emergence of a new unit of analysis for the study of contingent interactions: the forum as a whole.
\end{abstract}

Resumo. Este estudo tem como objetivo identificar e caracterizar contextos de aprendizagem favoráveis à implementação de interações contingentes em um fórum de discussão de um Curso de Formação Inicial de Tutores a Distância. A abordagem Etnográfica Interacional que norteou esta pesquisa estabelece quatro princípios operativos que guiam as decisões e ações do etnógrafo no campo de pesquisa. Tais princípios promoveram uma compreensão mais ampla sobre os contextos de aprendizagem no fórum. Os resultados do estudo apontam, principalmente: a fragilidade da representação da interação na ferramenta fórum investigada e o surgimento de uma nova unidade de análise para o estudo de interações contingentes: o fórum como um todo.

\section{Introdução}

Este artigo apresenta os resultados de uma pesquisa de pós-doutorado conduzida na Universidade da Califórnia, Santa Barbara (UCSB) durante o ano letivo de 2015-2016. O objetivo da pesquisa foi identificar e caracterizar a formação de contextos de aprendizagem que viabilizaram a manifestação de interações contingentes em um fórum de um curso de formação de professores, demonstrando como os participantes constroem conhecimento de maneira interativa por meio do discurso.

Para situar o público deste trabalho, é importante retornar às raízes do conceito de interações contingentes. Entende-se por interações contingentes processos de comunicação mais diretamente associados a engajamento e aprendizagem, baseados em um contexto que valorize práticas reflexivas, críticas e construtivas. Nesse tipo de troca comunicativa, há um esforço por parte dos interlocutores em construírem um discurso que favoreça o desenvolvimento de seus conhecimentos, por meio do esforço coletivo em comunicar mensagens com valor agregado, de fácil interpretação e cujo conteúdo 
desencadeie entre os leitores processos interativos contínuos e voltados para o aprendizado coletivo [David 2010].

$\mathrm{O}$ interesse por estudar os processos interacionais mediados pelas Tecnologias de Informação e Comunicação (TDIC) surgiu da experiência desenvolvida por uma das autoras deste trabalho no Instituto UFC Virtual da Universidade Federal do Ceará integrante do Sistema Universidade Aberta do Brasil (UAB). Ofertando cursos na modalidade a distância desde 2005, este Instituto possui seu próprio ambiente virtual de aprendizagem: o SOLAR (https://solar.virtual.ufc.br/).

A ferramenta Fórum do SOLAR possui atualmente diversos recursos de apoio ao professor, tais como: ferramentas avaliativas e para controle de frequência de alunos, duas formas de visualização de mensagens (em lista e em árvore, com sete níveis hierárquicos, e textos sem limites de caracteres), ferramenta SpeechToText (para ditar conteúdos), editor de fórmulas matemáticas, possibilidade de notificação de novas mensagens no e-mail do usuário e dois tipos de filtro (visualização de posts de discussões em que o usuário fez parte e uma tela com o resumo da atuação no fórum).

Não obstante, o volume de informações com as quais os professores de cursos online lidam em seu cotidiano ao mediar fóruns de discussão, seja pelo SOLAR ou por qualquer outro ambiente virtual, cresce bastante em turmas com mais de 20 alunos matriculados, tendo em vista a necessidade de avaliar-lhes o aprendizado e dar-lhes feedbacks regulares sobre as suas participações. O SOLAR ainda não dispõe de ferramentas de inteligência artificial. Além disso, os formatos de visualização das mensagens em lista e em árvore não favorecem uma boa análise para a tomada de decisões por parte dos professores. Em geral, o desenvolvimento de rotinas que contemplem tais demandas recorrem a estudos das áreas de Engenharia de Software, Inteligência Artificial ou Interação Humano-Computador (IHC).

Esta pesquisa pretende apresentar a Etnografia Interacional como uma lógica de investigação aplicada ao estudo da troca de mensagens por meio da Ferramenta Fórum do SOLAR em um curso de formação inicial de tutores, evidenciando a importância da visualização de contextos de aprendizagem para a emergência de interações contingentes. Inicialmente, são apresentados os fundamentos da Etnografia Interacional e seus conceitos-chave. Em seguida, contextos de aprendizagem são definidos compondo a base referencial do estudo para a análise das interações. Na sequência, o presente estudo é descrito, conforme os quatro princípios operativos da Etnografia Interacional. Ao final, os resultados são discutidos e as conclusões, apresentadas.

\section{A Etnografia Interacional como uma Lógica de Investigação}

A Etnografia Interacional (Interactional Ethnography) [Green and Dixon 1993] consiste em uma perspectiva teórica e metodológica para o desenvolvimento de investigações empíricas que busquem compreender os processos de aprendizagem imersos em possibilidades coletivas. Trata-se de uma lógica de pesquisa que integra perspectivas etnográficas e análise do discurso no estudo da dinâmica da vida em sala de aula.

Os três pressupostos da Etnografia como uma lógica de investigação, mencionados por Green et al. (2005) são: a) Etnografia como o estudo da cultura; b) Etnografia envolvendo uma perspectiva contrastiva; e, c) Etnografia envolvendo uma perspectiva holística.

Para as autoras, o estudo de fenômenos ou práticas culturais das vidas diárias dos grupos sociais investigados é central nesta abordagem etnográfica, tendo em vista promover uma compreensão sobre os padrões de comportamento e ação social dos membros desses grupos. Assim, o etnógrafo busca dar visibilidade às práticas culturais invisíveis inerentes ao contexto estudado (perspectiva êmica), com base em sua própria história intelectual e na lógica de investigação por ele adotada. 
De acordo com Castanheira (2004), a cultura deve ser compreendida como um sistema de significados dinâmico e compartilhado. Tal sistema é aprendido, revisto, mantido e definido no contexto em que as pessoas interagem. Dessa forma, a cultura não constitui um conjunto de significados antecipadamente definidos, mas é reconstruída localmente em espaços sociais particulares. A sala de aula é, portanto, um desses espaços, contendo uma cultura própria a ser explorada.

Outro conceito central no estudo da cultura de um grupo, dentro da abordagem etnográfica é a linguagem. Trata-se de um processo de interação que depende dos repertórios linguísticos que os membros trazem para a sala de aula. Vista de outra maneira, é também um sistema de discurso e ação social, constituído por meio das ações e interações entre professores e alunos.

Sobre os conceitos de linguagem e cultura, o antropólogo Michael Agar [Agar 2006] propõe uma síntese dos dois, por ele denominada linguacultura (languaculture). Para este teórico, cada mundo social possui sua linguacultura e os etnógrafos trazem a sua própria (LC1) para lerem e interpretarem a linguacultura daqueles em cujos mundos eles estão entrando (LC2). Para o autor, ao indagar-se sobre o que está acontecendo em relação ao fenômeno estudado, diferenças culturais tornam-se visíveis e é neste momento que a cultura acontece. Diferentes combinações LC1/LC2 geram pontos ricos para exploração e análise.

De acordo com Agar (2006), um ponto rico em uma pesquisa etnográfica acontece em situações que levem o etnógrafo a se questionar sobre o que está acontecendo naquele momento dentro do cenário de investigação, o que gerou o fenômeno em questão e que conhecimentos são necessários para iluminar esse fenômeno. Trata-se de conflitos nos quadros de referência ou quebras de enquadre (frame clashes) que criam um potencial para a exploração de conhecimentos culturais privilegiados. Tornam-se, portanto, âncoras para análises posteriores.

Por sua vez, os casos expressivos (telling cases) são apontados por Mitchell (1984) como os resultados de análises sobre um indivíduo ou um grupo social por longos períodos de tempo. Os eventos de um caso expressivo podem estar associados a quaisquer níveis da organização social (sociedade como um todo, comunidade, família ou indivíduo) e são construídos não apenas em função de eventos, mas também em função do tempo e dos atores envolvidos. Podem, portanto, reportar diferentes contextos de análise.

\section{Definindo contexto}

Contextos são ambientes constituídos interacionalmente, imersos no tempo e no espaço, que podem ser alternados de momento a momento. Corroborando com McDermott (1976), para quem as pessoas em interação tornam-se contexto umas para as outras, na Etnografia Interacional contextos sociais são definições compartilhadas e ratificadas de uma situação e em ações sociais das pessoas com base nessas definições. Dessa maneira, os contextos estão constantemente mudando e são constituídos mutuamente, mediante a definição de situações estabelecidas por meio de processos discursivos e interpretativos entre os participantes de um grupo.

De acordo com esses estudiosos, quando a questão do contexto é levantada, geralmente se argumenta que o evento focal não pode ser entendido, interpretado adequadamente ou descrito de maneira relevante, a menos que se olhe além do próprio evento para outros fenômenos (por exemplo, cenário cultural, situação de fala, premissas compartilhadas em segundo plano) nos quais o evento é incorporado ou, alternativamente, os recursos da própria conversa invocam premissas específicas em segundo plano relevantes para a organização da interação subsequente. "O contexto é, portanto, um quadro que envolve o evento que está sendo examinado e fornece recursos para sua interpretação apropriada" [Goodwin and Duranti 1992, p. 3]. 
Situações sociais constituem contextos nos quais as pessoas são agentes dinâmicos de contexto umas para com as outras. Nesse sentido, um elemento central com o qual os interlocutores precisam lidar é a participação das outras pessoas. Cada pessoa é um agente ativo com suas particularidades que são trazidas para o cenário da conversa. Essa complexidade do processo de interação enfatiza os desafios para a análise de eventos.

\subsection{Contextos de Aprendizagem}

Salas de aula são organizações sociais específicas, com demandas particulares de comunicação. Para Green and Wallat (1981) tais organizações sociais são contextos, vistos como "entidades socialmente ativas construídas por alunos e professores, ao se envolverem em interações sociais em sala de aula para atingir objetivos educacionais específicos" (p. 176). Contextos são diferentes de aulas e mudam quando os participantes se envolvem nos processos de interação, e também para além dos limites das aulas.

Kumpulainen and Erstad (2017), organizadores de uma edição especial do International Journal of Educational Research (IJER, v. 84, 2017), reuniram artigos de uma comunidade internacional de pesquisadores discutindo sobre como se dá a aprendizagem em diferentes contextos. Cada artigo apresenta concepções específicas para os conceitos de aprendizagem, aprendizes e contextos de aprendizagem.

Como parte da coletânea, Rasmussen and Damşa (2017) discutem aprendizagem como construção de sentido e definem contextos de aprendizagem como uma combinação de atividades em ambientes físicos e virtuais, incluindo o acesso a conhecimentos, recursos e ferramentas distribuídas através desses diferentes ambientes. Preocupados em compreender como aspectos constitutivos do processo de construção de sentido geram experiências de aprendizado, os autores analisaram a emergência de trajetórias de participação e agrupamentos de recursos pelos participantes, descrevendo como eles se envolvem na construção de sentido (re)criando novas estabilidades de conhecimentos e práticas ao longo do tempo. Eles compreendem a aprendizagem, então, a partir de uma infinidade de fontes disponíveis e, o mais importante, como é feita a capitalização desse conhecimento em atividades e práticas futuras.

Green and Wallat (1979) propõem um Sistema Sociolinguístico de Análise Etnográfica para mapear as interações sociais nas salas de aula que "combinam as dimensões social e linguística da situação em estudo" (p. 181). O sistema compreende: (a) o mapeamento das conversas durante o processo educacional; (b) a identificação de contextos; e, (c) a identificação de regras de ação social (p. 162).

Segundo as autoras, antes da identificação de contextos e regras dentro de um grupo de estudantes em uma sala de aula, é necessário mapear a evolução da conversa instrucional. Esse processo de mapeamento inclui a representação da evolução sequencial da conversa, mensagem por mensagem, elaborada por professores e estudantes. Em outras palavras, é importante registrar como as pessoas interagem e agem sobre as mensagens umas das outras. O mapa estrutural mostra como os participantes se organizam dentro da situação de interação na sala de aula, definindo contextos e criando regras sociais.

O sistema sociolinguístico apresentado em Green and Wallat (1979, pp. 164167) para descrever unidades e estruturas de conversação define a conversação de acordo com: (1) A fonte da mensagem (Source): Professor ou Estudante; (2) A forma (Form): Pergunta, Resposta, Resposta Esperada e Resposta Inesperada; (3) As Estratégias (Strategies): Iniciar, Ignorar, Aceitar, Rejeitar, Continuar, Estender, Controlar, Reorientar e Repetir; (4) Os níveis de compreensão (Levels): Factual, Interpretativo e Aplicativo; (5) Os elos (Ties): Professor, Estudante, Materiais 
IX Congresso Brasileiro de Informática na Educação (CBIE 2020)

Anais do XXXI Simpósio Brasileiro de Informática na Educação (SBIE 2020)

Didáticos; e, (6) As Unidades de interação (Interaction Units): Resolvidas e Nãoresolvidas.

Este sistema permite identificar diferentes unidades de conversação (unidade de mensagem, de interação, de sequência instrucional, de fase e aula), como também constatar os vários contextos de aprendizagem que se formam. De acordo com as autoras, é ao final das unidades de fase (Phase Units) que os contextos de aprendizagem se modificam.

\section{O Estudo}

Esta pesquisa cumpre duplo propósito: apresentar a Etnografia Interacional à comunidade acadêmica da área de Informática Educativa como uma lógica de investigação para o estudo do potencial educacional de ferramentas tecnológicas. Entende-se que este propósito foi cumprido mediante as discussões teóricas apresentadas nas seções anteriores deste artigo. $\mathrm{O}$ segundo propósito do presente estudo, a ser contemplado nesta seção, é apresentar o uso da Etnografia Interacional na identificação e caracterização de contextos de aprendizagem em um fórum de discussão online de um curso de formação inicial para professores-tutores, o qual contou com 26 participantes mais o tutor-formador.

O curso teve como objetivo capacitar professores de diferentes áreas de conhecimento para atuarem como tutores no Sistema Universidade Aberta do Brasil na Universidade Federal do Ceará, durante o ano de 2010. Com uma carga-horária de 90 horas-aula, ministradas predominantemente a distância por meio do ambiente virtual SOLAR, o curso contou com 06 aulas versando sobre diferentes temáticas ligadas à formação de tutores. O universo da pesquisa foi o fórum da Aula 4, da turma de professores de Matemática, intitulado Ação Tutorial.

É importante ressaltar esse mesmo fórum de discussão já foi analisado anteriormente à luz de um sistema categorial, buscando-se observar seu potencial dialógico [Freire et al 2011]. Nesta pesquisa, o fórum foi revisitado e novamente analisado à luz da Etnografia Interacional, buscando-se caracterizar os contextos de aprendizagem que se formaram durante os debates, demonstrando: como os participantes (professores em formação) constroem conhecimento interacionalmente por meio do discurso. Investigaram-se os contextos de aprendizagem que se formaram durante o fórum e que favoreceram o engajamento em interações contingentes.

As etapas que guiaram o desenvolvimento desta pesquisa foram inspiradas nos quatro princípios operativos da Etnografia Interacional, apresentados em Green et al. (2012). Segundo esses autores, pelo menos quatro princípios guiam uma pesquisa etnográfica:

1. Entender a Etnografia como um sistema não-linear - adequa-se a contextos de investigação em que o fenômeno em foco não pode ser previsto, e sim, construído dinamicamente;

2. Evitar o etnocentrismo - o etnógrafo entra no cenário de investigação sem hipóteses ou preconcepções, como um sistema de categorias, por exemplo;

3. Identificar os limites dos eventos - dada a complexidade do fenômeno, em determinados momentos são feitas escolhas para observá-lo;

4. Construir conexões - os diferentes dados coletados durante a pesquisa, em entrevistas, diários de campo, documentos etc. são contrastados para a elaboração dos resultados.

A seguir, os procedimentos de análise serão descritos, seguindo-se esses quatro princípios operativos. Para cada princípio, será apresentado um caso expressivo [Castanheira 2004] (ou telling case) os quais evidenciarão a lógica de investigação que guiou este estudo. 
IX Congresso Brasileiro de Informática na Educação (CBIE 2020)

Anais do XXXI Simpósio Brasileiro de Informática na Educação (SBIE 2020)

\subsection{Caso Expressivo 1: Traçando as raízes históricas da investigação de contextos de aprendizagem no fórum de discussão}

O primeiro princípio da Etnografia Interacional a descreve como uma lógica abdutiva, ao buscar a melhor explicação para um determinado acontecimento, sendo guiada por processos recursivos e iterativos para a explicação de fenômenos culturais, práticas e significados anteriormente desconhecidos em relação ao objeto de investigação. A identificação do conhecimento cultural (perspectiva êmica) somente é possível quando o etnógrafo identifica uma quebra de enquadre (frame clash), que não pode ser compreendida sem uma exploração mais aprofundada. E foi o que ocorreu quanto ao estudo das interações contingentes com base no Sistema de Análise de Interações Contingentes (SAIC) apresentado em David (2010).

Estudos sobre interações contingentes já foram desenvolvidos em diferentes contextos de investigação [Lamy and Goodfellow 1999, David and Castro-Filho 2012]. O SAIC representou um avanço frente às pesquisas anteriores por conjugar categorias que consideram diferentes facetas da comunicação online. A unidade de análise do referido sistema é a mensagem individual do fórum de discussão e suas categorias podem ser utilizadas para avaliar se as mensagens trocadas entre os estudantes de cursos online concorrem para o aprendizado individual e coletivo. Trata-se de um sistema que, de maneira abrangente, busca identificar se o tipo de interação estabelecido entre os participantes de fato está contribuindo para o seu aprendizado ou se, doutra sorte, as mensagens trocadas estão se afastando dos objetivos do curso.

Não obstante, a despeito do potencial do SAIC em contribuir com uma compreensão mais aprofundada e multifacetada do conceito de interação contingente, sua densidade categorial tornou complexa sua aplicação manual. Um primeiro esforço na direção de uma implementação automatizada do sistema, mediante o uso de ontologias aplicadas à categoria Diálogo, revelou a fragilidade do mesmo em lidar com disciplinas específicas ligadas a distintos campos de conhecimento, tais como: Línguas Estrangeiras, Matemática e Ciências [David et al. 2015], pela necessidade de uma especificação linguística minuciosa de suas categorias.

As pesquisadoras se acharam, então, diante de uma quebra de enquadre (frame clash). Algumas questões foram posicionadas quanto ao levantamento dos marcadores linguísticos característicos das categorias do SAIC, haja vista a intenção de aplicá-lo a diferentes contextos, níveis e áreas de estudo. Considerando-se a abrangência dos cenários de atuação da Universidade Federal do Ceará na área de Educação a Distância (cursos de extensão, graduação e pós-graduação), voltados para diferentes campos de conhecimento, tais reflexões produziram as seguintes questões de ordem Ontológica, Metodológica e Epistemológica:

- Marcadores linguísticos são específicos para cada língua, pessoas e contextos. Como o SAIC poderá lidar com diferentes contextos de aprendizagem? (Questão ontológica)

- Como analisar o processo global de interação, levando-se em consideração mensagens individuais emitidas pelos participantes como unidades de análise? (Questão metodológica)

- É possível, por meio do SAIC, obter informações contextuais sobre o campo específico no qual a análise será desenvolvida? (Questão epistemológica)

Em busca de respostas para essas questões, as pesquisadoras passaram a explorar uma nova lógica de investigação para a análise do fenômeno da interação com vistas ao aprendizado: a Etnografia Interacional.

\subsection{Caso Expressivo 2: De um sistema categorial à análise etnográfica}

O segundo Caso Expressivo da lógica de investigação adotada na pesquisa foi inspirado no segundo princípio que rege a Etnografia Interacional [Green et al. 2012]: evitar o 
etnocentrismo. Esse princípio determina que os etnógrafos rompam com seus próprios pontos de vista, expectativas ou interpretações sobre o fenômeno em foco com o objetivo de capturar o conhecimento interno do grupo estudado (perspectiva êmica). Algumas das ações envolvidas nesse pilar da etnografia consiste em examinar os objetivos do grupo, os conhecimentos compartilhados, bem como as normas e expectativas construídas, papeis, relacionamentos, direitos e deveres.

O percurso de análise do fórum de discussão foi iniciado mediante a proposta da seguinte questão geral de investigação: Como o processo de interação se desenvolve com base em diferentes contextos que se criam em fóruns de discussão do Curso de Formação Inicial de Tutores a Distância da Universidade Federal do Ceará, de forma a estimular o aprendizado dos estudantes sobre o tema acordado para discussão e o alcance do objetivo de aprendizagem estabelecido?

A partir dessa questão geral, deu-se início a um processo iterativo de investigação que envolveu o posicionamento de questões específicas sobre o fórum, a seleção de dados representativos e eventos de análise. No primeiro ciclo de análise, buscou-se compreender que fatores influenciavam as regras de participação dos estudantes no fórum, a partir do que estava estabelecido na ementa do curso, nos materiais didáticos disponíveis no SOLAR, e no modelo de avaliação da aprendizagem. A partir daí uma nova questão específica foi proposta, seguida da seleção de novos dados representativos e da implementação de novos eventos de análise.

Uma imagem ilustrativa da lógica de investigação envolvida nesta pesquisa encontra-se representada na Figura 1, a seguir:

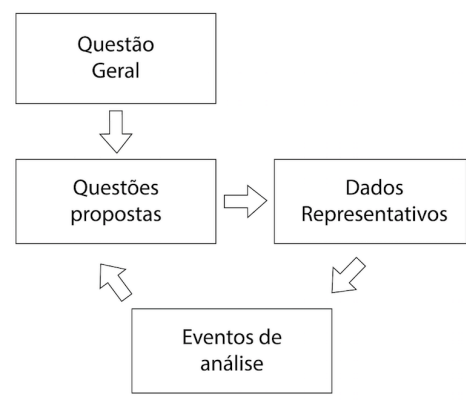

Figura 1. Imagem representativa da lógica de investigação da pesquisa

A seguir, um Caso Expressivo será descrito, demonstrando como a investigação de contextos de aprendizagem em fóruns de discussão à luz da Etnografia Interacional conduziu a uma mudança na unidade de análise da pesquisa.

\subsection{Caso Expressivo 3: Redefinindo a unidade de análise para o estudo dos contextos de aprendizagem no fórum de discussão}

Este caso expressivo se inspira no terceiro e no quarto Princípios Operativos da Etnografia Interacional: Identificar os Limites dos Eventos e Construir Conexões [Green et al. 2012]. Trata-se de um processo de tomada de decisão para a definição precisa acerca da unidade de análise do evento investigado, de tal forma que a descrição do mesmo seja o mais abrangente possível sobre o que está acontecendo nas atividades selecionadas, cenários ou grupos de participantes observados. Por sua vez, o processo de redefinição da unidade de análise só pode ser implementado mediante um contraste entre os dados coletados: linhas do tempo temáticas, mapas de eventos, além dos diferentes mecanismos de interação adotados pelos participantes. A aplicação do sistema sociolinguístico de Green and Wallat (1979) ao fórum foi central para a definição da nova unidade de análise do fórum de discussão para a identificação dos contextos de aprendizagem: o fórum como um todo. 
Para a identificação dos limites dos eventos que emergiram no fórum, as mensagens trocadas foram mapeadas em função de linhas do tempo (threads) temáticas, as quais chamaram a atenção das pesquisadoras para a forma como as mensagens da ferramenta Fórum da Plataforma SOLAR está organizada, sendo considerada inadequada à identificação de contextos de aprendizagem. No fórum em foco, foram identificadas 23 threads que se formaram espontaneamente, algumas vezes pelos estudantes e outras vezes pelo tutor. As linhas de discussão foram classificadas de acordo com um código (número), quantidade de mensagens trocadas, participante que a iniciou, período de duração e relação com a questão-chave proposta para o debate.

Foi feito um retorno à descrição e proposta do fórum para se identificar as threads efetivamente relacionadas ao tema principal. Juntamente com a descrição da questão-chave do fórum foi realizado um contraste de análises sobre as orientações para as atividades dadas aos estudantes nos materiais didáticos do curso, bem como em orientações dadas pelo tutor no decorrer das discussões. Das 23 threads do fórum, a princípio, apenas 08 demonstraram estar efetivamente relacionadas ao tema proposto para o debate: Ação Tutorial. Foram elas: T2, T5, T7, T13, T14, T17, T18 e T19. Neste momento, tomou-se a decisão de se fazer um zoom nessas threads para observar as ações linguísticas dos participantes e, assim, compreender como se formam os contextos de aprendizagem, objetivo desta investigação.

Esta etapa da análise se baseou no sistema sociolinguístico de Green and Wallat (1979, pp. 164-167) para descrever unidades e estruturas de conversação na investigação das threads. Como discutido na seção 3.1. deste artigo, o sistema descreve diversos aspectos da conversação, tendo sido aplicado às 295 mensagens do fórum, possibilitando a geração de gráficos por linha de discussão, sintetizando os resultados da descrição sociolinguística de forma a identificar os contextos de aprendizagem. Entre as dimensões analisadas é relevante destacar as Unidades de Interação, conforme tenham sido Resolvidas ou Não-resolvidas. Uma Unidade de Interação Resolvida, consiste em um agrupamento de mensagens relacionadas por tópico ou intenção, que atinge seu propósito geral. Por outro lado, Unidades de Interação Não-Resolvidas compreendem mensagens sem resposta que tenham sido ignoradas no percurso do debate.

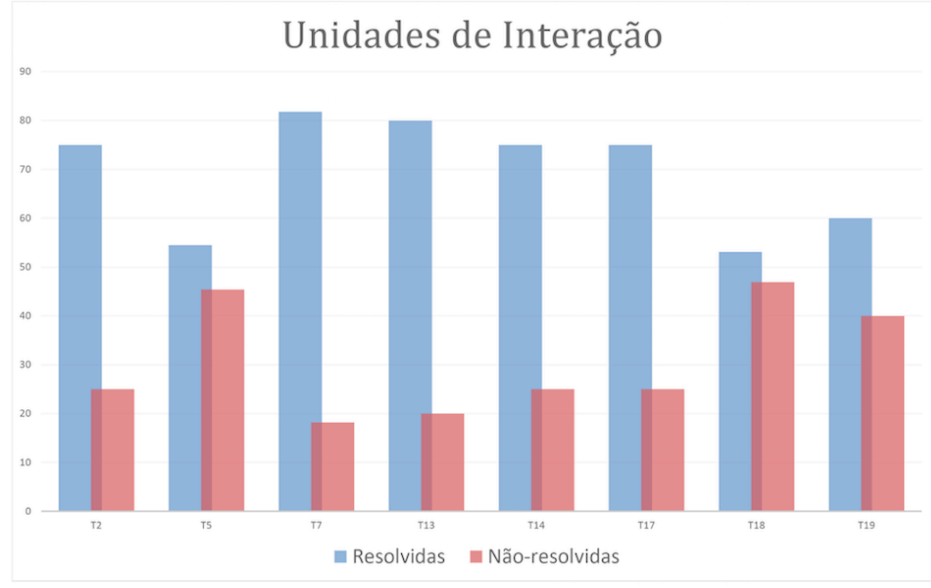

Gráfico 1. Análise das Threads do Fórum conforme a Resolução das Interações

O Gráfico 1 demonstra que a maior parte das threads apresentaram Unidades de Interação Não-Resolvidas, cujos temas foram complementados em outras threads, constatação feita mediante um novo ciclo de análise das threads anteriormente não incluídas na amostra (Figura 2). 


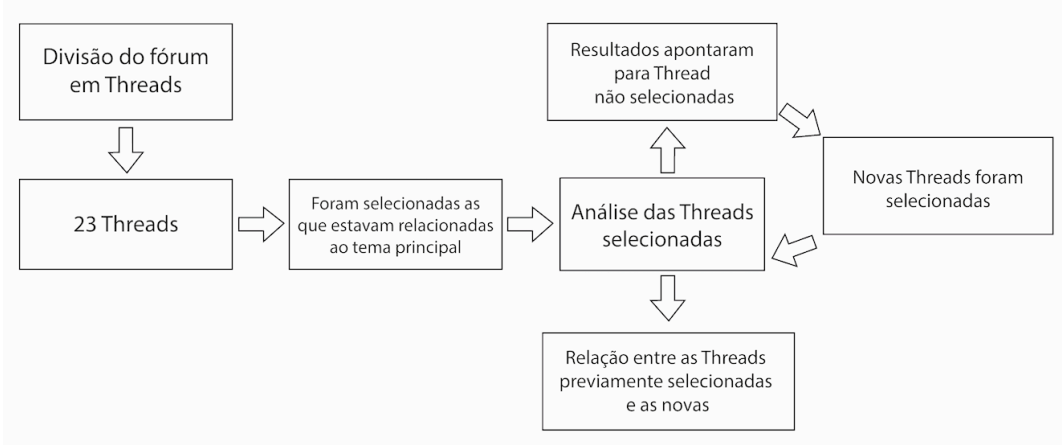

Figura 2. Fluxo de Análise das Linhas Temáticas das Threads do Fórum

A análise realizada por meio do sistema sociolinguístico de Green and Wallat (1979) para a identificação dos contextos de aprendizagem, requereu que fosse feito um retorno aos dados em busca de Unidades de Fase (Phase Units), isto é, de possíveis conexões entre threads. Uma primeira conexão encontrada foi entre as Threads 2 e 6 , esta última fora do grupo de threads inicialmente consideradas aderentes ao tema. Isto revelou que threads inicialmente consideradas fora do contexto de discussão (como era o caso da T6) na verdade estavam conectadas ao tema, algumas vezes complementado o conteúdo de outra thread.

Este Caso Expressivo demonstra, portanto, a inadequação da mensagem individual como unidade de análise para a investigação dos contextos de aprendizagem. Nesta perspectiva, percebeu-se a necessidade de se modificar a unidade de análise da presente investigação haja vista que mensagens individuais não dão conta de representar todos os aspectos envolvidos no fenômeno em investigação: contextos de aprendizagem.

\section{Conclusões}

A Etnografia Interacional foi apresentada neste artigo como uma abordagem metodológica para o estudo de contextos de aprendizagem em fóruns de discussão online. Trata-se de uma lógica de pesquisa de raízes antropológica cultural, sociolinguística e etnográfica comunicacional, tendo em vista contribuir com o estudo dos fenômenos de aprendizagem mediados pelas TDIC.

$\mathrm{O}$ estudo constatou a necessidade de que o pesquisador estabeleça uma coerência entre o fenômeno que se está investigando, no caso desta pesquisa, o processo de interação e a formação de contextos de aprendizagem, com a abordagem metodológica adotada para a compreensão deste fenômeno. Sistemas categoriais nem sempre dão conta de explicar o fenômeno da interação social por ser ativamente construído, de modo que estruturas ou regras pré-estabelecidas não parecem adequadas. Além disso, enfatiza-se aqui a importância de levar em consideração o contexto em que o processo de interação ocorre, a fim de entender como a linguagem funciona para produzir os múltiplos resultados da conversação.

A análise do fórum de discussão em apreço, à luz da Etnografia Interacional, apontou: 1) a existência uma conexão temática entre as threads do fórum de discussão, mediante o início das reflexões sobre um tema em um espaço do fórum e a conclusão do mesmo em outro espaço/thread; 2) nesse sentido, a análise de mensagens individuais mostrou-se inadequada para explicar o fenômeno das interações contingentes e dos contextos de aprendizagem, sendo importante ampliar a unidade de análise da mensagem individual para o fórum de discussão como um todo; 3) ficou constatado também que a representação atual da interação social na ferramenta Fórum do AVA SOLAR mostrou-se limitada para mapear a complexidade da construção de conhecimento em cenários educacionais online. 


\section{Agradecimentos}

As autoras agradecem à CAPES, à Fulbright e ao CNPq pelo financiamento desta pesquisa.

\section{Referências}

Agar, M. (2006). "An Ethnography By Any Other Name", In: Forum Qualitative Sozialforschung / Forum: Qualitative Social Research, [S.1.], v. 7, n. 4.

Castanheira, M. L. (2004). Aprendizagem contextualizada: discurso e inclusão na sala de aula, Belo Horizonte: CEALE/Autêntica.

David, P. B. (2010). Interações contingentes em ambientes virtuais de aprendizagem. Tese (Doutorado em Educação) - Universidade Federal do Ceará, Faculdade de Educação, Programa de Pós-Graduação em Educação Brasileira, Fortaleza-CE.

David, P. B.; Castro-Filho, J. A. (2012). "Sistema de Análise de Interações Contingentes: Uma Contribuição para Práticas Interativas em Cursos a Distância". Revista e-Curriculum (PUCSP), v. 8, p. 1-28.

David, P. B.; Trajano de Lima, E.; Mendes, F. A. G. (2015). “Uma Ontologia de Domínio para a Análise do Diálogo Freireano em Fóruns de Discussão da Educação a Distância". In: Anais do XXVI Simpósio Brasileiro de Informática na Educação (SBIE), IV Congresso Brasileiro de Informática na Educação e X Conferência Latino-Americana de Objetos e Tecnologias de Aprendizagem, Maceió-ẢL.

Freire, R. S., David, P. B. and Oliveira, F. K. (2011) "Dialogicidade na Formação Online de Professores de Matemática", In: Anais do XXII Simpósio Brasileiro de Informática na Educação, Aracaju-SE.

Goodwin, C. and Duranti, A. (1992). Rethinking Context: an Introduction. In: Rethinking Context: Languagem as an interactive phenomenon. Alessandro Duranti and Charles Goodwin, eds., Interaction and gramar, pp. 1-42. Cambridge: Cambridge University Press.

Green, J. and Dixon, C. (1993) "Talking Knowledge into Being: Discursive and Social Practices in Classrooms", Linguistics and Education, v.5, n. 3-4, p. 231-239.

Green, J. L., Dixon, C. N. and Zaharlick, A. (2005) "A etnografia como uma lógica de investigação”, In: Educação em Revista, Belo Horizonte, v. 42, p. 13-79.

Green, J. and Wallat, C. (1979) "What is an Instructional Context? An Exploratory Analysis of Conversational Shifts Across Time". Ablex Publishing Corporation: Norwood, New Jersey.

Green, J. and Wallat, C. (1981) "Mapping instructional conversations: A sociolinguistic ethnography", In: Ethnography and Language in Educational Settings, v. 5, p. 161-205.

Kumpulainen, K. and Erstad, Ola. (2017) “(Re)Searching learning across contexts: Conceptual, methodological and empirical explorations", In: International Journal of Educational Research (IJER), v. 84, p. 55-57.

Lamy, M.; Goodfellow, R. (1999). "Reflective Conversation" in the Virtual Language Classroom. Language Learning \& Tecnology, v.2, n.2, p. 43-61, Disponível em: <http://llt.msu.edu/vol2num2/article2>. Acesso em: ago. 2020.

McDermott, R. (1976) "Kids Make Sense: an Ethnographic Account of the Interactional Managementof Success and Failure", In: One First-Grade Classroom. Ph.D. Dissertation, Stanford.

Mitchell, D.C. (1984) "An evaluation of subject-paced reading tasks and other methods for investigating immediate processes in Reading", In: New methods in reading comprehension research. Hillsdale, Erlbaum. Kieras, D. \& Just, M.A. (Eds.)

Rasmussen, I.; Damşa, C. I. (2017). "Heterochrony through moment-to-moment interaction: A micro-analytical exploration of learning as sense making with multiple resources", In: International Journal of Educational Research (IJER), v. 84, p. 79-89. 